$$
\lambda=h \frac{m^{2}}{m^{2}-4}
$$

wo $h$ eine Constante, $m$ die laufenden Zahlen von $m=3$ angefangen bedeuten. Ich kann mir nicht versagen, die Genauigkeit der Formel für die Hagenbach'sche Rechnung hier hervorzuheben. Für die Beobachtungen von Cornu ist $h=364.542$, für die von Müller und Kempf $h=$ $3^{64.6205}$ gesetzt.

\begin{tabular}{|c|c|c|c|c|c|}
\hline $\begin{array}{l}\text { Hydro } \\
\text { genlini }\end{array}$ & $m$ & Cornu & berechnet & $\begin{array}{l}\text { Müller und } \\
\text { Kempf }\end{array}$ & berechnet \\
\hline $\begin{array}{l}\alpha \\
\beta\end{array}$ & $\begin{array}{l}3 \\
4\end{array}$ & $\begin{array}{l}656.2 \mathrm{I} \\
486.07\end{array}$ & $\begin{array}{l}656.18 \\
486.06\end{array}$ & $\begin{array}{l}656.314 \\
486.164\end{array}$ & $\begin{array}{l}656.3 \text { I } 7 \\
486.16 \text { I }\end{array}$ \\
\hline$\gamma$ & $\begin{array}{l}5 \\
6\end{array}$ & $\begin{array}{l}433.95 \\
410.12\end{array}$ & $\begin{array}{l}433.9^{8} \\
4 \text { I } 0.11\end{array}$ & $\begin{array}{l}434.071 \\
410.198\end{array}$ & $\begin{array}{l}434.072 \\
410.198\end{array}$ \\
\hline $\begin{array}{l}\varepsilon \\
\zeta\end{array}$ & $\begin{array}{l}7 \\
8\end{array}$ & $\begin{array}{l}396.92 \\
388.8 \mathrm{I}\end{array}$ & $\begin{array}{l}396.95 \\
388.84\end{array}$ & 396.879 & 397.031 \\
\hline$\eta$ & 9 & 383.49 & $3^{8} 3.4^{8}$ & & \\
\hline$\theta$ & 10 & 379.73 & 379.73 & & \\
\hline$\iota$ & I I & 376.99 & 377.00 & & \\
\hline$x$ & I 2 & 375.02 & $374.9^{6}$ & & \\
\hline$\lambda$ & 13 & $373.4^{1}$ & $373.3^{8}$ & & \\
\hline$\mu$ & I 4 & 372.11 & 372.34 & & \\
\hline$v$ & I 5 & 37 I.1 2 & 371.14 & & \\
\hline
\end{tabular}

Die Formel hat sich schon bei mehreren Elementen bewährt; ist sie allgemein, so liesse sich daraus $R$ der Gleichung (14) bestimmen, indem man hätte:

$$
R=\frac{2 x^{2} m^{2}-x^{4}}{m^{4}}, \quad \mu_{0}^{2}=--h^{2}
$$

wo $x$ irgend eine Constante - ganze Zahl - bedeutet, welche die Ordnungszahl der Linie $\lambda=\infty$ angiebt. Wäre aber $h^{2}=+\mu_{0}{ }^{2}$, so wäre $x$ einfach die Zahl der auftretenden Linien. Darüber liegen noch keine Erfahrungen vor.

Nimmt man an, die Chromosphäre der Sonne bestände vornehmlich aus Wasserstoff, so wäre für denselben $\boldsymbol{\mu}=$ 0.5361 , während $\mu_{0}=0.3645$ ist. Die Temperatur der Sonne müsste noch etwa $3 / 2$ mal so hoch sein, damit Dissociation des Wasserstoffs eintreten könnte.

Ich muss mir versagen, auch nur anzudeuten, welchen Einfluss die vorgetragene Theorie auf die in der Astrophysik anzuwendende Wärmetheorie ausübt, und welche Folgerungen für die Molekularphysik erwachsen. Gelingt es, die Balmer' sche Gleichung als allgemein darzustellen, und das scheint aus der Grundgleichung wenigstens formell möglich, so haben wir in der zweckmässigen Beobachtung des Spektrums für die Molekulardynamik das gewonnen, was der Astronom in der Ortsbestimmung der Gestirne findet, und auch dem Astrophysiker ist die Möglichkeit geboten, sein System auf eine so feste Grundlage - die mechanische Wärmetheorie - zu bauen, wie sie die Astronomie in der Mechanik besitzt, denn statt der unzugänglichen Zustandselemente Druck und Temperatur haben wir die augenfälligen Variabeln des Spektrums, welche auch die Gleichungen und Definitionen der Hauptsätze der Wärmetheorie beträchtlich zu vereinfachen scheinen.

Sternwarte Kis Kartal, Ungarn, ${ }_{1887}$ August 28 .

Ring Micrometer Observations of Winnecke's Comet $1886 \mathrm{VI}$

\begin{tabular}{|c|c|c|c|c|c|c|c|c|c|c|c|c|}
\hline I 886 & Nashv, M.T. & $\Delta \alpha$ & & $\Delta \delta$ & $\mathrm{Cp}$. & & $\alpha$ app. & & app & & Red.ad l. app. & $*$ \\
\hline Sept. I 7 & $7^{\mathrm{h}} 43^{\mathrm{m}} 37^{\mathrm{s}}$ & $-0^{m} 54^{s} \cdot 15$ & - & 6'26"I & 4 & $15^{\mathrm{h}}$ & $8^{\mathrm{m}} 20^{5} \cdot 3 \mathrm{I}$ & $-20^{\circ}$ & $37^{\circ}$ & I 6"4 & $+I^{s} \cdot 3 I+0.4$ & 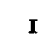 \\
\hline 18 & $733 \quad 59$ & $-3 \times 6.97$ & & $5 \quad 19.8$ & 3 & & - & & 一 & & $+1.32+0.4$ & 2 \\
\hline 18 & 73359 & -521.82 & $+I$ & $0 \quad 19.2$ & 3 & & - & & - & & $+1.32+0.4$ & 3 \\
\hline 20 & $\begin{array}{lll}727 & 45\end{array}$ & $-7 \quad 10.43$ & - & I 8.0 & 3 & I 5 & $23 \quad 5^{6.98}$ & -22 & 46 & 50.1 & $+1.40+1.0$ & 4 \\
\hline 20 & 72745 & $\begin{array}{ll}-8 & 43.67\end{array}$ & & $\circ 18.0$ & 3 & I 5 & 2356.8 I & -22 & 46 & 50.9 & $+1.40+1.0$ & 5 \\
\hline 2 I & 71950 & -4 I 5. & - & $2 \quad 32.3$ & 4 & & - & & - & & $+1.40+0.9$ & 6 \\
\hline 22 & 72629 & -244.16 & - & 932.1 & 5 & 15 & $34 \quad 48.64$ & -22 & I I & $3^{x} \cdot 5$ & $+1.42+0.8$ & 7 \\
\hline 22 & 72629 & $-4 \quad 16.93$ & +1 & $0 \quad 2 . I$ & 5 & 15 & $34 \quad 47.29$ & -22 & I I & 20.0 & $+1.42+0.8$ & 8 \\
\hline 23 & $\begin{array}{lll}7 & 23 & 4\end{array}$ & $-0 \quad 53.15$ & - & I I 7.1 & 6 & & - & & - & & $+1.43+0.9$ & 9 \\
\hline Oct. I 6 & $7 \quad 1 \quad 32$ & -237.55 & - & 916.0 & 2 & & - & & - & & $+1.90+4.3$ & Io \\
\hline v. 15 & 737 & - I 6.42 & + & I 43.1 & 3 & & - & & - & & $+2.04+9.3$ & I I \\
\hline 18 & 728 & +346.20 & -1 & $55 \cdot \mathrm{I}$ & 3 & & 一 & & - & & $+2.01+9.6$ & I 2 \\
\hline 18 & $\begin{array}{lll}7 & 28 & 3\end{array}$ & +059.67 & + & 348.5 & 3 & 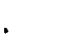 & - & & - & & $+2.02+9.5$ & I3 \\
\hline 25 & $731 \quad 55$ & +24.62 & + & $3 \quad 53.9$ & 4 & $2 \mathrm{I}$ & $55 \quad 3.27$ & -29 & 35 & 51.7 & $+2.03+10.1$ & I 4 \\
\hline
\end{tabular}

made at the Vanderbilt University Observatory, Nashville, Tenn., by E. E. Barnard.

Sept. I 7. V g b M to probably a faint nucleus. - Sept. I 8. Brightish with glimpses of a stellar nucleus. Sept. 2o. About $\mathbf{I}^{\prime}$ diam. Round $\mathrm{g} b \mathbf{M}$ to a small ill defined nucleus. The comet of $9^{1} / 2^{\mathrm{m}}$ or $\mathbf{r} \mathrm{O}^{\mathrm{m}}$. - Sept. 22 . Brightish, fully $I^{\prime}$ diam. with ill-defined nucleus of $9^{\mathrm{m}}$ or less. The light of the comet is whitish. - Oct. 16. Average size; $\mathrm{vg} \mathrm{b} \mathrm{M} \mathrm{faintish.} \mathrm{-} \mathrm{Nov.} \mathrm{I5.} \mathrm{Not} \mathrm{very} \mathrm{faint,} \mathrm{but} \mathrm{hard} \mathrm{to} \mathrm{observe} \mathrm{on} \mathrm{account} \mathrm{of} \mathrm{bright} \mathrm{star} \mathrm{in} \mathrm{field.} \mathrm{-} \mathrm{Nov.} \mathrm{I} 8$. Faintish and largish; v g b M. - Nov. 25. Faint but largish; v g b M. 
Mean Places of Comparison Stars for 1886.0.

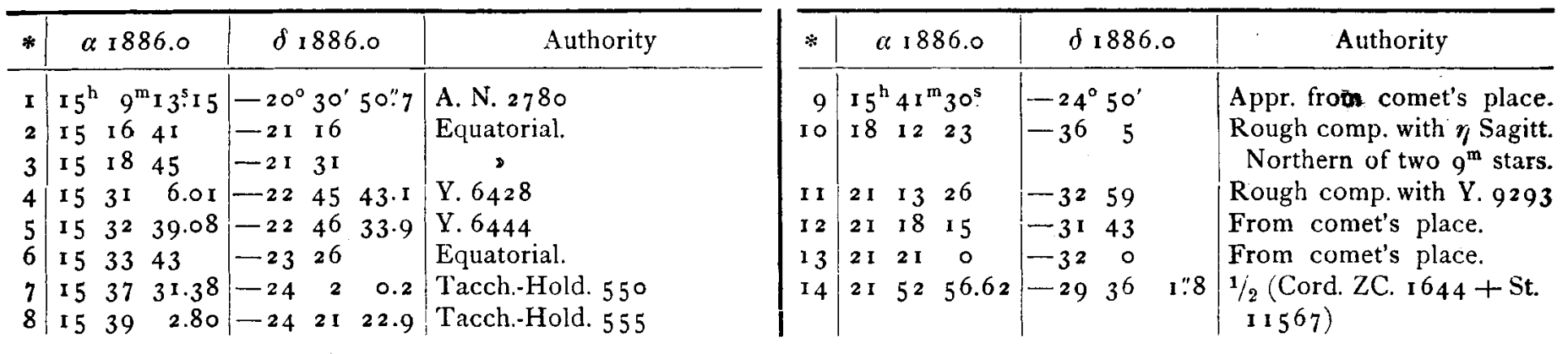

It is singular that no ephemeris of this comet was published during this apparition, for I have seen none. If such had been published more observations of the comet could have been made. I mistook it several times for a new comet having swept it up whilst comet seeking, and remained in considerable suspense, not knowing certainly that it was Winnecke's comet. - The observations are corrected for refraction.

Nashville, Tenn., 1887 August.

\section{Observations of Comets}

made at the Royal Observatory, Cape of Good Hope.

(Communicated by D. Gill LL.D., F.R.S., Her Majesty's Astronomer.)

\begin{tabular}{|c|c|c|c|c|c|c|c|c|c|c|}
\hline 1887 & Cape M. T. & $\Delta \alpha$ & $\Delta \delta$ & Cp. & $\alpha$ app. & $\log p \cdot A$ & $\delta$ app. & $\log p . A$ & Red. ad 1.app. & * \\
\hline
\end{tabular}

Comet I886 IX.

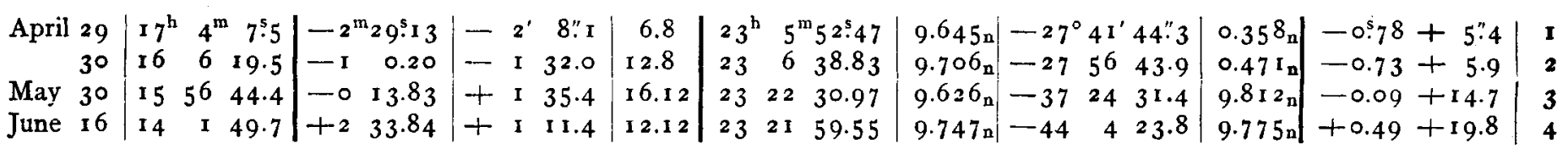

These observations were made by Mr. Finlay with the filar micrometer of the 7 inch Equatoreal; they are corrected for refraction.

The comet was small and faint, with a slight tendency to condensation towards the centre.

May 30. Occasional glimpses of a star-like nucleus $=$ about 1 I mag.

June 16 . Comet faint, about $1 / 2^{\prime}$ in diameter, with slight condensation towards the centre.

$$
\text { Comet } 1887 \text { IV (Barnard May } 12 \text { ). }
$$

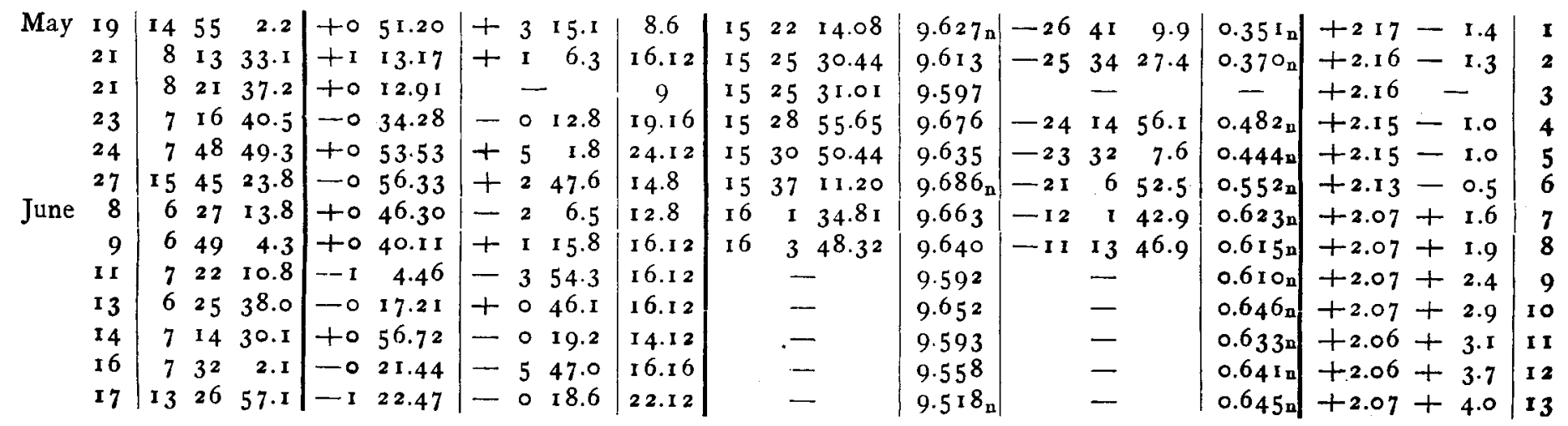

All the observations have been made by Mr. Finlay with the 7 inch Equatoreal and Repsold filar micrometer. May 19. Comet is small and round, equal in brightness to a star of 1o mag.; not difficult to observe.

May 21. Hazy sky; Comet and stars faint.

May 23, June 8. Comet shows a nucleus of about i I mag. 